\title{
The Choice of the Endpoint to Assess the Efficacy or Effectiveness in Advanced or Metastatic Cancer Tumors
}

Takayoshi Kiba

Division of Modern Medical Technology, Institute for Clinical Research, National Hospital Organization Kure Medical Center, Kure, 737-0023

\begin{abstract}
It is important to investigate whether other clinical endpoints, such as response rate, disease stabilization rate, or progression free survival could replace overall survival as the primary endpoint for the patients with advanced or metastatic cancer. Before a surrogate end point can replace a so-called 'true' end point of interest, it must be formally validated, a process that has caused considerable controversy in the past two decades. The aim of this review manuscript is to discuss some of the limitations encountered when survival is used as the primary study end point for evaluating the efficacy or effectiveness in phase II or III trials for advanced or metastatic cancer tumors.
\end{abstract}

Keywords: Clinical trial; Endpoint; Overall survival; Response rate; Progression free survival; Efficacy; Effectiveness

Abbreviations: EMEA: European Agency for the Evaluation of Medical Products; FDA: Food and Drug Administration; ICH: International Conference on Harmonisation of Technical Requirements for Registration of Pharmaceuticals for Human Use; OS: Overall Survival; PFS: Progression Free Survival

\section{Introduction}

The Food and Drug Administration (FDA) is undertaking a project to evaluate potential endpoints for cancer drug approval. Endpoints will be examined for the most common cancers. For each cancer, FDA will hold public workshops to identify important issues, and these issues will be discussed in meetings of the Oncologic Drugs Advisory Committee. Therefore, FDA provides a comparison of endpoints in cancer drug approval (U.S. Department of Health and Human Service). Moreover, many issues relating to the proper analysis of efficacy endpoints are addressed in the International Conference on Harmonisation of Technical Requirements for Registration of Pharmaceuticals for Human Use (ICH) guideline for industry E9 Statistical Principles for Clinical Trials. In general, progression free survival (PFS) is useful at the time of therapy or drug development because it is not convoluted with patient specific characteristics, specific disease related health issues or subsequent therapies complications. To the contrary, overall survival (OS) is more important to patient, and this endpoint should be taken for the basis when several treatment choices are discussed with the patient. Before a surrogate end point can replace a so-called 'true' end point of interest, it must be formally validated, a process that has caused considerable controversy in the past two decades. The U.S. FDA provided guidance documents during 1980s that indicated that efficacy should be demonstrated by prolongation of life, improved healthrelated quality of life, or an established surrogate for at least one of these. Interestingly, the weight of the evidence provided by a survival analysis is substantially different on the two sides of the Atlantic. Indeed, the US FDA considers survival benefit the cornerstone for approval of new anticancer drugs in the United States, whereas the European Agency for the Evaluation of Medical Products (EMEA) accepts a prolongation in time to progression as a primary requirement for new drug registration in the European Union [1,2]. The aim of this review manuscript is to discuss some of the limitations encountered when survival is used as the primary study end point for evaluating the efficacy or effectiveness in phase II or III trials for advanced cancer tumors.

\section{Response rate or progression free survival as the endpoint of phase II}

Table 1 shows that main primary endpoints used in advanced or metastatic various types of cancers of phase II study. After treatment with active agents, response rates or PFS intervals often vary widely among phase II studies because of variation in patient selection and response measurement. However, single arm phase II studies of combination regimens using tumor shrinkage endpoints or of single agents using PFS endpoints are problematic. Whereas tumors rarely shrink spontaneously, PFS times often vary widely among patients and determining whether a drug has extended PFS requires the measurement of PFS times for a comparison group of patients who did not receive the drug. However, interpretation of single arm phase II study results is different when a new drug is used combination with other agents and when PFS is used as the endpoint rather than tumor shrinkage. Moreover, it was reported that a meta-analysis of randomized trials in advanced colorectal cancer, in which treatment had a significant effect on both response and survival, also failed to validate response as a surrogate for survival [3].

Burzykowski et al. [4] conducted a meta-analysis on the basis of individual patient data from 11 randomized trials including 3953 patients and comparing an anthracycline (alone or in combination) with a taxane as first-line therapy for metastatic disease of breast cancers. The results indicated that PFS is not a good surrogate for overall survival in this setting because of an only moderate correction between treatment effects on these two end points. On the other hand, taking a

Corresponding author: Takayoshi Kiba, MD, PhD, Division of Modern Medical Technology, Institute for Clinical Research, National Hospital Organization Kure Medical Center, Kure, 737-0023, Hiroshima, JAPAN, Tel: 81_4-7137-3737; E-mail: kibat@kure-nh.go.jp

Received March 09, 2011; Accepted September 08, 2011; Published September 11,2011

Citation: Kiba T (2011) The Choice of the Endpoint to Assess the Efficacy or Effectiveness in Advanced or Metastatic Cancer Tumors. J Cancer Sci Ther 3 154-157. doi:10.4172/1948-5956.1000079

Copyright: (c) 2011 Kiba T. This is an open-access article distributed under the terms of the Creative Commons Attribution License, which permits unrestricted use, distribution, and reproduction in any medium, provided the original author and source are credited. 
trial-level approach, Miksad et al. [5] came to a different conclusion, after finding that the hazard ratios (HR) for PFS were significantly correlated with HR for overall survival in trials of anthracyclines and taxanes, albeit with only modest explained variances.

The response rate encompasses complete responses and partial response and does not include a measure of stable disease. Response rate is considered direct evidence of pharmacologic activity of the drug. Unlike OS and PFS, which must be evaluated in randomized trials, response rates can be accurately assessed using a single-arm trial. Response rate has also been a surrogate of OS in only a few malignancies. In those malignancies, where increased response rate did not result in survival advantage, it was assumed that tumor shrinkage may lead to a decrease in tumor-associated symptoms, and hence, to clinical benefit. This is a biologically plausible assumption, although it has been rarely supported by convincing evidence. Although the FDA has used response rate as the basis for regular and accelerated approvals, it was reported that the agency acknowledges that tumor responses do not necessarily equate with clinical benefit from delay in tumor progression [6].

Does evidence suggest that response rate fully captures the effect of treatment on survival, when conducting a Cox regression analysis using response rate as a time varying covariate [7], or when generating survival curves by tumor response using the landmark method? [8]. Although these statistical analyses provide evidence concerning the plausibility that response rate is a useful surrogate end point, one must use considerable caution in interpreting these results. The limitation is that these analyses only address whether response rate is capturing the net effect of treatment on survival. In such setting, treatment might also be providing additional beneficial effects on survival through mechanisms other than indication of response, but these additional benefits may be offset by the treatment's unintended adverse effects on survival that are not captured by response rate. It also follows that it is unwise to generalize the relationship between effects on response rate and effects on survival found with one class of agents to other classes. The another limitation is that the effect of treatment might not be a result of response rate but, indeed, a result of a causal mechanism that is corrected with the response rate, such as prevention of long-term worsening of tumor burden if that is induced in predominantly the same patients who experience tumor response. The other limitation is the substantial variability in parameter estimates that is inherent with these methods. This has an important impact on the reliability of these methods, particularly in setting such as those chosen by Bruzzi et al. [9] where treatment has a small effect on survival. The landmark analyses also have the limitation as result of the risk of bias arising from missing data, exclusion of early deaths, and dependence on strong assumptions regarding independent censoring that are unlikely to hold, and as a result of the compromised interpretability when there is large variability in response time [9].

Grothey et al. [10] considered PFS and the percentage of patients experiencing tumor control as the most appropriate end points for trial design in advanced colorectal cancer. Several investigators have attempted to assess the correlation between treatment effects on OS and on potential surrogate endpoints in advanced breast cancer [9]. In the past, PFS was not used as primary surrogate endpoint for market authorization. However, PFS is a lucrative endpoint because it captures events of progressions and death, both of which are important, plausible endpoints in cancer therapy. Due to this definition, stable disease is captured as a benefit of therapy. It requires a smaller number of patients enrolled in clinical trials, and shorter follow-up when compared with survival studies. Moreover PFS is not affected by crossover or subsequent therapies, and events of progression are

Table 1: Main primary endpoints used in advanced or metastatic various types of cancers.

\begin{tabular}{|c|c|c|}
\hline & Phase II & Phase III \\
\hline Non-small cell lung cancer & response rate, progression free survival & overall survival, progression free survival \\
\hline Small-cell lung cancer & response rate, progression free survival & overall survival, progression free survival \\
\hline Mesothelioma & response rate & overall survival \\
\hline Breast cancer & response rate, progression free survival & overall survival, progression free survival \\
\hline Nasopharyngeal carcinoma & response rate, time to progression & overall survival, progression free survival \\
\hline Thyroid cancer & response rate & progression free survival \\
\hline Esophageal cancer & response rate & overall survival, progression free survival \\
\hline Gastric cancer & response rate & overall survival \\
\hline Colon rectal cancer & response rate, progression free survival & overall survival, progression free survival \\
\hline Pancreatic cancer & response rate, progression free survival, overall survival & overall survival \\
\hline Hepatocellular carcinoma & response rate, progression free survival & overall survival, time to progression \\
\hline Biliary tract Cancer & response rate, progression free survival & overall survival \\
\hline Cervical cancer & response rate & overall survival, progression free survival \\
\hline Endometrial Cancer & response rate, progression free survival & overall survival, recurrence free survival \\
\hline Ovarian cancer & response rate, progression free survival & overall survival, progression free survival \\
\hline Prostate Cancer & time to PSA progression, progression free survival & overall survival, progression free survival \\
\hline Renal cell carcinoma & response rate, progression free survival & overall survival, progression free survival \\
\hline Glioblastoma & response rate, progression free survival & overall survival, progression free survival \\
\hline Melanoma & $\begin{array}{l}\text { response rate, relapse free survival, disease } \\
\text { stabilization rate }\end{array}$ & overall survival, progression free survival \\
\hline
\end{tabular}


based on objective quantitative assessment. PFS is also an attractive end point for clinical trials because it is available earlier than OS, is less influenced than OS by competing causes of death, and is not influenced by second-line treatment. However, the use of PFS is not without problems in the context of clinical trials. In contrast to the objectivity of OS, the ascertainment of disease progression is potentially subject to measurement error and bias. PFS may also be contrasted with objective response as a potential surrogate end point for OS. Although responses in individual patients correlate with OS at the individual level in both breast [11] and colorectal cancer [12], treatment benefits in terms of response rates do not reliably predict treatment benefit in terms of PFS or OS. This issue becomes even more important with the targeted agents currently available, for which long-term benefits have sometimes been seen despite the lack of significantly improved response rates [13]. However, the fact that PFS has been shown to be an acceptable surrogate for OS in absence of effective second-line therapies provides partial support to the view that PFS is a desirable end point to use in future clinical trials. The endpoint should accurately assess the efficacy of the drug being evaluated, and the endpoint and the trial design should minimize potential bias. A'Hern et al. [11] analyzed the correction between response rates and OS and found a statistically significant relationship between these two end points by weighted linear regression. Moreover, it was recently reported that that PFS in advanced gastric cancer is strongly correlated with OS at the individual level, but treatment effects on PFS are only moderately correlated with treatment effects on OS [14].

\section{Overall survival as the endpoint of phase III}

Table 1 also shows that main primary endpoints used in advanced or metastatic various types of cancers of phase III study. OS remains the gold standard for the demonstration of clinical benefit. This endpoint is unambiguous and is not subject to investigator interpretation. OS is a direct clinical benefit to patients, and assessment can be calculated to the day. An improvement in OS is a direct clinical benefit to patients. An analysis of OS requires larger patient numbers and longer follow-up than other endpoints. OS is considered a precise and easily measured endpoint that is clinically meaningful and not subject to bias. Although the demonstration of longer OS may be considered a preferred endpoint, the use of this endpoint has limitations [15]. Survival analysis may be confounded by subsequent therapies. Limitations of survival analyses include long follow-up periods in large trials, and the effect of subsequent therapies may confound the analysis of survival. Burzykowski et al. [4] reported that OS should be viewed as the endpoint of choice to assess the efficacy of new treatments in advanced breast cancer.

Since effective salvage therapies currently exist and may compensate for less-active first-line therapies, OS may no longer be the most appropriate primary efficacy end point in the first-line therapy of advanced colorectal cancer [10]. In the majority of currently reported phase III trials in advanced disease, survival is not the primary study endpoint, although survival analysis is regularly performed and always represents a secondary objective. The undeniable advantage of survival as a study endpoint is that it represents the ultimate clinical benefit for the patient, provided that quality of life is not compromised. Moreover, many recently reported trials in advanced colorectal cancer, lacked power to detect a statistically significant increase in OS, even in the presence of other benefits [16]. Continuing demonstration of OS gain in advanced cancer is likely to become increasingly rare in the near future, once the use of effective agents becomes more widespread.

\section{Overall survival as the endpoint of phase II}

In the previous reported phase II study, it was reported that the targeted patients resistant to both antracycline and taxane had highly dismal disease, whose 1 year survival was estimated $<33 \%$ [17], thereby OS was chosen for the primary endpoint. Some investigators reported that OS should be viewed as the endpoint of choice to assess the efficacy of new treatments in phase II trial of the advanced breast cancer [18].

\section{Conclusions}

In this review article, the author has discussed some of the limitations encountered when survival is used as the primary study end point for evaluating the efficacy or effectiveness in phase II or III trials for advanced or metastatic cancer tumors. Throughout oncology, recently, many biomarkers have been evaluated. Example include direct measures of the tumor burden process, such as response rate or PFS, or inherently less reliable indirect measure, such as carcinoembryonic antigen or prostate-specific antigen. Although it is a direct measure of the tumor burden process, response rate could underestimate treatment effects on clinical end points, such as survival, by failing to adequately capture the magnitude, breadth, and in particular, the duration of effects on tumor burden. Conversely, response rate could overestimate impact on survival or other clinical end points if response is brief or if this measure fails to capture unintended harmful mechanisms of action of treatment. Various authors have proposed meta-analytic approaches, arguing that a large body of data from individual patients were required for validation of end points [3]. They suggested that the association between the surrogate and true end points should be assessed after adjustment for the treatment effect, thus introducing the concept of individual level surrogacy. They also proposed that a surrogate end point be assessed both 'individual level' and at the 'true level' for its ability to predict the effect of treatment on the true end point, after observation of the treatment effect on the surrogate. Of note, the trial level correlation is mathematically independent of the individual level correction (at least for normally distributed end point), which is somewhat counterintuitive but implies that a claim of surrogacy requires stronger condition than a mere correlation between the surrogate and the true end point.

\section{Disclosure}

The author declare no conflict of interest.

\section{References}

1. U.S. Department of Health and Human Services. Food and Drug Administration. Center for Drug Evaluation and Research. Center for Biologics Evaluation and Research. Guidance for industry: Clinical Trials Endpoints for the Approval of cancer Drugs and Biologics.

2. European Agency for the Evaluation of Medicinal Products (EMEA). Note for guidance on evaluation of anticancer medical products in man.

3. Buyse M, Piedbois P, Piedbois Y, Carison RW (2000) Meta-analysis: methods strengths, and weakness. Oncology (Williston Park) 14: 437-443.

4. Burzykowski T, Buyse M, Piccart-Gebhart MJ, Sledge G, Carmichael J, et al. (2008) Evaluation of tumor response, disease control, progression-free survival, and time to progression as potential surrogate end points in metastatic breast cancer. J Clin Oncol 20: 1987-1992.

5. Miksad RA, Zietemann V, Gothe R, Schwarzer R, Conrads-Frank A, et al (2008) Progression-free survival as a surrogate endpoint in advanced breast cancer. Int J Technol Assess Health Care 24: 371-383.

6. Johnson JR, Williams G, Pazdur R (2003) End points and United States Food and Drug Administration approval of oncology drugs. J Clin Oncol 21: 14041411. 
Citation: Kiba T (2011) The Choice of the Endpoint to Assess the Efficacy or Effectiveness in Advanced or Metastatic Cancer Tumors. J Cancer Sc Ther 3: 154-157. doi:10.4172/1948-5956.1000079

7. Lin DY, Fleming TR, De Gruttola V. (1997) Estimating the proportion of treatment effect explained by a surrogate marker. Stat Med 16: 1515-1527.

8. Simon R, Makuch RW (1984) A non-parametric graphical representation of the relationship between survival and the occurrence of an event: application to responder versus non-responder bias. Stat Med 3: 35-44

9. Bruzzi P, Del Mastro L, Sormani MP, Bastholt L, Danova M, et al. (2005) Objective response to chemotherapy as a potential surrogate end point of survival in metastatic breast cancer patients. J Clin Oncol 23: 5117-5125.

10. Grothey A, Hedrick EE, Mass RD, Sarkar S, Suzuki S, et al. (2008) Responseindependent survival benefit in metastatic colorectal cancer: a comparative analysis of N9741 and AVF2107. J Clin Oncol 26: 183-189.

11. A'Hern RP, Ebbs SR, Baum MB (1988) Does chemotherapy improve survival in advanced breast cancer? A statistical overview. Br J Cancer 57: 615-618.

12. Buyse M, Thirion P, Carlson RW, Burzykowski T, Molenberghs G, et al. (2000) Relation between tumour response to first-line chemotherapy and survival in advanced colorectal cancer: a meta-analysis. Meta-Analysis Group in Cancer. Lancet 356: 373-378

13. Moore MJ, Goldstein D, Hamm J, Figer A, Hecht JR, et al. (2007) Erlotinib plus gemcitabine compared with gemcitabine alone in patients with advanced pancreatic cancer: a phase III trial of the National Cancer Institute of Canada Clinical Trials Group. J Clin Oncol 25: 1960-1966.

14. Shitara K, Burzykowski T, on behalf of GASTRIC project (2011) Progressionfree survival as surrogate endpoint of overall survival in patients with advanced/ recurrent gastric cancer: Individual patient data analysis on 4,102 patients from 20 randomized trials. J Clin Oncol 29: (suppl; abstr 4095).

15. Sargent D (2006) General and statistical hierarchy of appropriate biologic endpoints. Oncology (Williston Park) 20: 5-9.

16. Di Leo A, Bleiberg H, Buyse M (2003) Overall survival is not a realistic end point for clinical trials of new drugs in advanced solid tumors: a critical assessment based on recently reported phase III trials in colorectal and breast cancer. $J$ Clin Oncol 21: 2045-2047.

17. Ando M, Watanabe T, Nagata K, Narabayashi M, Adachi I, et al. (2001) Efficacy of docetaxel $60 \mathrm{mg} / \mathrm{m} 2$ in patients with metastatic breast cancer according to the status of anthracycline resistance. J Clin Oncol 19: 336-342.

18. Ishida T, Kiba T, Takeda M, Matsuyama K, Teramukai S, et al. (2009) Phase II study of capecitabine and trastuzumab combination chemotherapy in patients with HER2 overexpressing metastatic breast cancers resistant to both anthracyclines and taxanes. Cancer Chemother Pharmacol 64: 361-369. 